\title{
Urinary tract infections in Romanian patients with diabetes: prevalence, etiology, and risk factors
}

\author{
This article was published in the following Dove Press journal: \\ Therapeutics and Clinical Risk Management \\ 16 December 2016 \\ Number of times this article has been viewed
}

\author{
Teodora Chițăă \\ Bogdan Timar ${ }^{1,2}$ \\ Delia Muntean ${ }^{1,2}$ \\ Luminița Bădițoiu' ${ }^{1,3}$ \\ Florin Horhat ${ }^{1,2}$ \\ Elena Hogea' \\ Roxana Moldovan ${ }^{1,3}$ \\ Romulus Timar ${ }^{1,2}$ \\ Monica Licker ${ }^{1,2}$ \\ 'Victor Babes University of Medicine \\ and Pharmacy Timisoara, ${ }^{2}$ Pius Brinzeu \\ Emergency Hospital, ${ }^{3}$ Regional Centre \\ of Public Health, Timisoara, Romania
}

Correspondence: Bogdan Timar Victor Babes University of Medicine and Pharmacy Timisoara, 2 Eftimie Murgu,

Timisoara 30004I, Romania

Tel +40 74I 528093

Fax +40 256462856

Email timar.bogdan@umft.ro
Aim: Patients with diabetes mellitus (DM) have an increased risk of infections, especially urinary tract infections (UTIs). The aim of this study was to assess the prevalence and etiology of UTIs and identify the risk factors for their development in patients with DM.

Patients and methods: In this retrospective, noninterventional study, the medical records of 2,465 adult patients with DM who were hospitalized in a Diabetes Clinic were reviewed. Data regarding the presence of UTI and possible associated risk factors were collected and their possible relation was analyzed. The study protocol and procedures were approved by the Ethics Committee of Timișoara Emergency Hospital. All data were collected and analyzed using SPSS v.17 statistical software.

Results: The prevalence of UTIs in patients with DM was 12.0\% (297 cases), being higher in females than in males and higher in patients with type 2 DM compared with patients with type $1 \mathrm{DM}$. In univariate logistic regression analysis, risk factors associated with UTIs were female gender, age, type $2 \mathrm{DM}$, longer duration of DM, and the presence of chronic kidney disease and coronary artery disease. Multivariate analysis identified age, duration of DM, and metabolic control (hemoglobin A1c levels) as independent risk factors for UTIs. The gramnegative bacilli from the Enterobacteriaceae family were predominant, with Escherichia coli being the most frequent of them (70.4\%).

Conclusion: UTIs are a frequent condition associated with DM. It is necessary to improve the care and the screening of UTIs in patients with DM to prevent the occurrence of possible associated severe renal complications.

Keywords: urinary tract infections, diabetes mellitus, epidemiology, incidence

\section{Introduction}

Patients with diabetes mellitus (DM) are more prone to develop infections compared with the general population, ${ }^{1}$ with urinary tract infections (UTIs) being among the most commonly encountered. ${ }^{2}$

It has been estimated that UTIs account for 7 million hospital visits per year, along with 1 million visits to the emergency department, in the United States, ${ }^{2}$ involving an estimated annual cost of $\sim$ US $\$ 1.6$ billion to the health care system. ${ }^{3}$

Several factors contribute to an increased infection risk in patients with DM: defects in the host immune defense mechanisms (such as impaired neutrophil function, decreased T-cell-mediated immune response, low levels of prostaglandin $\mathrm{E}$, thromboxane B2, leukotriene B4), ${ }^{4}$ incomplete bladder emptying due to autonomic neuropathy, and poor metabolic control. ${ }^{5,6}$ A higher glucose concentration in the urine allows urinary colonization by pathogenic microorganisms. ${ }^{4,5}$

Various types of UTIs may develop in patients with DM: asymptomatic bacteriuria (ASB), lower UTI (cystitis), upper UTI (pyelonephritis), and severe urosepsis, requiring submit your manuscript Dovepress ff
Therapeutics and Clinical Risk Management 2017:13 I-7

(c) (i) (8) 2017 Chiţă et al. This work is published and licensed by Dove Medical Press Limited. The full terms of this license are available at https://www.dovepress.com/terms.ph (c) ${ }_{\mathrm{BY}} \mathrm{NC}$ and incorporate the Creative Commons Attribution - Non Commercial (unported, v3.0) License (http://creativecommons.org/licenses/by-n/3.0/). By accessing the work you hereby accept the Terms. Non-commercial uses of the work are permitted without any further permission from Dove Medical Press Limited, provided the work is properly attributed. For permission for commercial use of this work, please see paragraphs 4.2 and 5 of our Terms (https://www.dovepress.com/terms.php). 
careful management in this category of patients. ${ }^{7,8}$ Rare complications of UTIs, including renal papillary necrosis, emphysematous cystitis and pyelonephritis, and intrarenal and perinephric abscesses, occur more often and with greater severity in patients with DM. ${ }^{7,8}$

Escherichia coli and other Enterobacteriaceae are the most commonly isolated uropathogens both in females and in males with DM, similar to the general population. However, UTIs caused by unusual and antibiotic-resistant uropathogens as well as fungal UTIs appear to be more frequently reported among patients with DM. ${ }^{7-9}$

No studies assessing the risk factors for UTIs among patients with DM have been conducted in Romania. Therefore, the present study has been undertaken to evaluate the prevalence and spectrum of etiologic agents of UTIs and to identify the risk factors for UTIs in a population of patients with DM from Western Romania.

\section{Materials and methods}

\section{Study design, setting, and population}

A hospital-based, retrospective, observational study that enrolled 2,465 Caucasian patients with DM was conducted. They were admitted in the Clinic of Diabetes, Nutrition and Metabolic Diseases at the Emergency Clinical County Hospital, Timişoara, Romania, from January 2011 to December 2012. This institution is a 1,173-bed tertiary-care university-affiliated teaching hospital providing health care services for the Western region of Romania.

To be included in the study, the patients had to have type 1 or type $2 \mathrm{DM}$ and had to be aged $\geq 18$ years. Patients with DM and positive urine cultures, without any other possible infection site, were included in the UTI-positive group. Both symptomatic and asymptomatic UTIs were included. The control group consisted of patients with DM, without any diagnosed infection in the last month prior to the hospital admission.

The following categories of patients were excluded from the study: patients without DM, those with prediabetes, females with gestational DM, nursing home or long-term care facility residents, patients undergoing antimicrobial treatment for a previously diagnosed UTI, patients with regular hemodialysis clinic visits, and those with infections other than UTIs. The study flow diagram is presented in Figure 1.

In the Clinic of Diabetes, Nutrition and Metabolic Diseases, urine cultures were performed in all patients suspected of UTIs: symptoms suggesting UTIs (dysuria, urgency, frequency of urination, suprapubic pain or tenderness, fever)

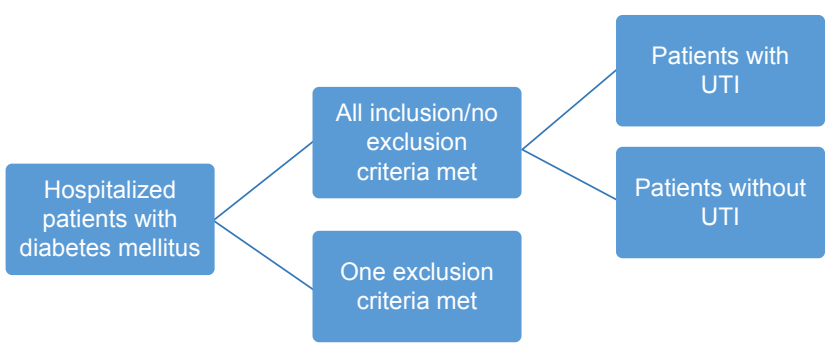

Figure I Study flow diagram.

Abbreviation: UTI, urinary tract infection.

or urinalysis with the presence of nitrite, leukocyte esterase, or $>5$ white blood cells per high-power field.

This study was approved by the Timişoara Emergency Hospital Ethics Committee; all patients provided written informed consent prior to their hospital admission for all the procedures performed during hospitalization, including the tests performed for the present study and for the use of their data for scientific research publications.

\section{Study protocol and definitions}

Hospital records of all the enrolled patients were reviewed by two independent reviewers, who were trained in data abstraction procedure. The collected data included demographics, anthropometric measurements, type and duration of DM, presence of comorbidities commonly associated with DM (retinopathy, chronic kidney disease, coronary artery disease, cerebrovascular disease, peripheral artery disease, neuropathy), UTI diagnoses, etiology and antimicrobial susceptibility testing (AST), renal function (assessed using serum creatinine and estimated glomerular filtration rate [eGFR], calculated using Chronic Kidney Disease Epidemiology Collaboration creatinine equation), and the quality of the glycemic control (evaluated using the hemoglobin $\mathrm{A} 1 \mathrm{c}[\mathrm{HbA} 1 \mathrm{c}])$. Significant bacteriuria was defined as the presence of $\geq 10^{5}$ colony-forming units per milliliter of urine. A symptomatic UTI was defined as the presence of bacteria in a patient with fever of urinary symptoms. ASB was defined as bacteriuria without fever of urinary symptoms. The diagnosis of UTIs (cystitis, pyelonephritis, and ASB) was established solely by the treating physician. Only the first episode of the positive urine culture per patient was included in the analysis.

\section{Identification of germs and AST}

Identification of germs was generally done according to morphological, cultural, and biochemical characteristics. The AST was assessed by performing the minimum 
inhibitory concentration, with automated reading and classification into resistance phenotypes by use of the Vitek 2 Compact analyzer (bioMérieux), according to the Clinical Laboratory and Standards Institute criteria. ${ }^{10}$

\section{Statistical analysis}

Data were collected and analyzed using IBM SPSS version 17 software suite (SPSS, Chicago, IL, USA) and are presented as medians and interquartile range for continuous variables without Gaussian distribution, mean \pm standard deviation for continuous variables with Gaussian distribution, and number of cases and percent from total for categorical variables. To assess the significance of the differences between groups, Mann-Whitney $U$ tests (medians, non-Gaussian populations) and Student's $t$-tests (means, Gaussian populations) were used. Chi-square analysis and Fisher's exact test were used to test categorical variables. Odds ratio (OR) was calculated to determine the risk of occurrence of UTIs after exposure or nonexposure to a certain factor. Logistic regression model was used to analyze the risk factors in cases in which the exposure was quantified through continuous, nondichotomous variables. The selection of predictors in the model followed a stepwise consecutiveprospective acceptance process (predictors being accepted at a significance level of $P \leq 0.1$ ), the chosen model being the one that best explained the occurrence of UTIs (maximum Nagelkerke coefficient) and the minimum deviation from the theoretical model (estimated by the method described by Hosmer and Lemeshow). A $P$-value $<0.05$ was considered statistically significant.

\section{Results}

\section{Baseline characteristics of the study sample}

The baseline characteristics of the patients are presented in Table 1.

\section{Prevalence of UTIs}

The prevalence of UTIs in the study group was $12.0 \%$ (297 cases). Regarding the difference between genders, $17.7 \%$ of females and $5.2 \%$ of males developed a UTI $(P<0.001)$. The prevalence of UTIs was higher in patients with type 2 DM compared to patients with type $1 \mathrm{DM}(12.6 \%$ vs $7.7 \% ; P=0.014)$. Out of the total number of UTIs, 40 cases $(13.5 \%)$ were classified as lower UTIs, 33 (11.1\%) upper UTIs, and 224 (75.4\%) were ASB. The prevalence of UTIs by type of infection was $1.4 \%$ lower UTIs, $1.6 \%$ upper UTIs,
Table I Baseline characteristics of the study sample

\begin{tabular}{|c|c|}
\hline Number of patients & 2,465 \\
\hline Age $(\text { years })^{\mathrm{a}}$ & $6 \mathrm{I}(15)$ \\
\hline Male $^{b}$ & $\mathrm{I}, 106(44.9)$ \\
\hline Urban area ${ }^{b}$ & $\mathrm{I}, 640(66.5)$ \\
\hline Body mass index $\left(\mathrm{kg} / \mathrm{m}^{2}\right)^{\mathrm{c}}$ & $29.9 \pm 6.6$ \\
\hline \multicolumn{2}{|l|}{ Diabetes type ${ }^{b}$} \\
\hline Type I & $298(12.1)$ \\
\hline Type 2 & $2,167(87.9)$ \\
\hline Diabetes duration (years) ${ }^{\mathrm{a}}$ & $8(10)$ \\
\hline Serum creatinine $(\mathrm{mg} / \mathrm{dL})^{c}$ & $1.0 \pm 0.7$ \\
\hline Glycated hemoglobin level (percentage points) ${ }^{c}$ & $8.9 \pm 2.0$ \\
\hline Hypertensive patients ${ }^{\mathrm{b}}$ & $1,912(77.6)$ \\
\hline \multicolumn{2}{|l|}{ Comorbidities of the urinary tract ${ }^{\mathrm{b}}$} \\
\hline Kidney stones & $184(7.5)$ \\
\hline Malformations of the urinary tract & $20(0.8)$ \\
\hline Benign prostatic hyperplasia & $223(9.1)$ \\
\hline Urinary catheterization & $7(0.3)$ \\
\hline Tumors of the urinary tract & II (0.4) \\
\hline Cystocele & $62(2.5)$ \\
\hline Renal cyst & $90(3.7)$ \\
\hline Two or more associated factors & $14(0.6)$ \\
\hline \multicolumn{2}{|l|}{ Diabetes complications ${ }^{\mathrm{b}}$} \\
\hline \multicolumn{2}{|l|}{ Retinopathy } \\
\hline Mild nonproliferative & $450(18.3)$ \\
\hline Moderate nonproliferative & $69(2.8)$ \\
\hline Severe nonproliferative & $|3|(5.3)$ \\
\hline Proliferative & $125(5.1)$ \\
\hline Chronic kidney disease & $576(23.4)$ \\
\hline Coronary artery disease & $1,208(49.0)$ \\
\hline Cerebrovascular disease & $167(6.8)$ \\
\hline Peripheral artery disease & $410(16.6)$ \\
\hline \multicolumn{2}{|l|}{ Neuropathy } \\
\hline Somatic & I,656 (67.I) \\
\hline Autonomic & $49(2.0)$ \\
\hline Positive urine culture & $297(12.0)$ \\
\hline
\end{tabular}

Notes: ${ }^{2} C o n t i n u o u s$ variables without Gaussian distribution. Results are presented as median and (interquartile range). 'Dichotomous variables. Results are presented as number of individuals and (percentage of total). 'Continuous variables with Gaussian distribution. Results are presented as average \pm standard deviation.

and $9 \%$ ASB. The prevalence of different types of UTIs by diabetes type and by gender is illustrated in Table 2 .

\section{Factors associated with the presence of UTIs}

The presence of UTIs in patients with DM was associated with higher age (65 vs 59.8 years; $P<0.001$ ), longer duration of DM ( 11 vs 9.5 years; $P=0.003$ ), and decreased eGFR ( $70.5 \mathrm{vs} 80.1 \mathrm{~mL} / \mathrm{min} / 1.73 \mathrm{~m}^{2} ; P<0.001$ ). The presence of UTIs was also associated with a significantly increased prevalence of chronic kidney disease $(18.4 \%$ vs $10.1 \%$; $P<0.001)$ and coronary artery disease $(13.7 \%$ vs $10.4 \%$; $P=0.011)$. The body mass index value had no significant 
Table 2 Prevalence of different types of UTls by type of diabetes and by gender

\begin{tabular}{|c|c|c|c|c|c|c|}
\hline \multirow[t]{2}{*}{ Type of UTI } & \multicolumn{2}{|c|}{ Diabetes type } & \multirow[t]{2}{*}{$P$-value } & \multicolumn{2}{|l|}{ Gender } & \multirow[t]{2}{*}{$P$-value } \\
\hline & Type I & Type 2 & & Male & Female & \\
\hline Lower UTI & $5(1.7)$ & $35(1.6)$ & 0.897 & $8(0.8)$ & $32(2.4)$ & 0.003 \\
\hline Upper UTI & $3(1.0)$ & $30(1.4)$ & 0.793 & $7(0.6)$ & $26(1.9)$ & 0.052 \\
\hline Asymptomatic bacteriuria & $15(5)$ & $209(9.6)$ & 0.013 & $42(3.8)$ & $182(13.4)$ & 0.046 \\
\hline Total & $23(7.7)$ & $274(12.6)$ & $<0.001$ & $57(5.2)$ & $240(17.7)$ & $<0.001$ \\
\hline
\end{tabular}

Note: Data are expressed as n (\%), unless otherwise indicated.

Abbreviation: UTI, urinary tract infection.

impact on the incidence of UTIs $(P=0.437)$. Between the studied parameters of the metabolic control $(\mathrm{HbAlc})$ and the presence of UTIs, no statistically significant association $(P=0.405)$ was found (Table 3$)$.

\section{Risk factors for UTls \\ Univariate analysis}

The univariate logistic regression model, which investigated the influence that different predictors had on the

Table 3 Comparison between patients with diabetes mellitus with/without UTI

\begin{tabular}{|c|c|c|c|}
\hline Host factor & $\begin{array}{l}\text { UTI- } \\
\text { negative }\end{array}$ & $\begin{array}{l}\text { UTI- } \\
\text { positive }\end{array}$ & $P$-value \\
\hline Age (years) ${ }^{b}$ & $59.8 \pm 12.1$ & $65 \pm 11.3$ & $<0.001$ \\
\hline Gender $^{\mathrm{a}}$ & & & $<0.001$ \\
\hline Male & $\mathrm{I}, 049(94.8)$ & $57(5.2)$ & \\
\hline Female & $1,119(82.3)$ & $240(17.7)$ & \\
\hline Body mass index $\left(\mathrm{kg} / \mathrm{m}^{2}\right)^{\mathrm{b}}$ & $30.0 \pm 6.5$ & $29.7 \pm 6.9$ & 0.437 \\
\hline Diabetes type ${ }^{a}$ & & & 0.014 \\
\hline 1 & $275(92.3)$ & $23(7.7)$ & \\
\hline 2 & I,893 (87.4) & $274(12.6)$ & \\
\hline Diabetes duration (years) ${ }^{\mathrm{b}}$ & $9.5 \pm 8.3$ & $11.0 \pm 9.1$ & 0.003 \\
\hline \multicolumn{4}{|l|}{ Diabetes complications $\mathrm{s}^{\mathrm{a}}$} \\
\hline Retinopathy & & & 0.127 \\
\hline Mild nonproliferative & $394(87.6)$ & $56(12.4)$ & \\
\hline Moderate nonproliferative & $59(85.5)$ & $10(14.5)$ & \\
\hline Severe nonproliferative & $107(81.7)$ & $24(18.3)$ & \\
\hline Proliferative & $107(85.6)$ & $18(14.4)$ & \\
\hline Chronic kidney disease & $470(81.6)$ & $106(18.4)$ & $<0.001$ \\
\hline Coronary artery disease & $1,042(86.3)$ & $166(13.7)$ & 0.011 \\
\hline Cerebrovascular disease & |4| (84.4) & $26(15.6)$ & 0.148 \\
\hline Peripheral artery disease & $360(87.8)$ & $50(12.2)$ & 0.921 \\
\hline \multicolumn{4}{|l|}{ Neuropathy } \\
\hline Somatic & $\mathrm{I}, 445(87.3)$ & $211(12.7)$ & 0.131 \\
\hline Autonomic & $46(93.9)$ & $3(6.1)$ & 0.198 \\
\hline Blood urea nitrogen $(\mathrm{mg} / \mathrm{dL})^{\mathrm{b}}$ & $43.8 \pm 26.7$ & $52.2 \pm 36.8$ & $<0.001$ \\
\hline Serum creatinine $(\mathrm{mg} / \mathrm{dL})^{\mathrm{b}}$ & $1.0 \pm 0.7$ & $1.1 \pm 0.9$ & 0.028 \\
\hline $\begin{array}{l}\text { Estimated glomerular filtration } \\
\text { rate }\left(\mathrm{mL} / \mathrm{min} / 1.73 \mathrm{~m}^{2}\right)^{\mathrm{b}}\end{array}$ & $80.1 \pm 30$ & $70.5 \pm 32.3$ & $<0.001$ \\
\hline Glycated hemoglobin level (\%) & $8.9 \pm 2.0$ & $9.0 \pm 1.9$ & 0.405 \\
\hline
\end{tabular}

Notes: a Dichotomous variables. Results are presented as number of individuals and (percentage from total). ' ${ }^{\circ}$ Continuous variables with Gaussian distribution. Results are presented as average \pm standard deviation.

Abbreviation: UTI, urinary tract infection. risk of occurrence of UTIs, found significant impact of age (OR $=1.04-$ per 1 -year increase; $P<0.001)$, diabetes duration (OR $=1.02$ - per 1-year increase; $P=0.003$ ), eGFR $(\mathrm{OR}=0.90-$ per $10 \mathrm{~mL} / \mathrm{min}$ increase; $P<0.001)$, serum creatinine $(\mathrm{OR}=1.18-$ per $1 \mathrm{mg} / \mathrm{dL}$ increase; $P=0.011)$, and blood urea nitrogen (BUN) $(\mathrm{OR}=1.10-$ per $10 \mathrm{mg} / \mathrm{dL}$ increase; $P<0.001)$. The HbA1c levels did not significantly influence the risk of occurrence of UTIs. Other factors that influenced the risk of developing UTIs were female gender ( $\mathrm{OR}=3.94 ; P<0.001)$, type $2 \mathrm{DM}(\mathrm{OR}=1.73 ; P<0.014)$, and the presence of chronic kidney disease $(\mathrm{OR}=1.42$; $P=0.033)$ and coronary artery disease $(\mathrm{OR}=1.36 ; P=0.011)$. No association was evident between UTIs and other analyzed predictors (retinopathy, cerebrovascular disease, neuropathy) (Table 4).

\section{Multivariate analysis}

Multivariate analysis identified age ( $\mathrm{OR}=1.04 ; P=0.001$ ), duration of DM (OR $=1.03 ; P=0.036)$, BUN $(\mathrm{OR}=1.10$; $P=0.023)$, and HbA1c levels ( $\mathrm{OR}=1.13 ; P=0.017)$ as significant, independent risk factors for UTIs (Table 5).

Table 4 Risk factors associated with urinary tract infections univariate analysis

\begin{tabular}{llll}
\hline Predictor & Unit & Odds ratio & P-value \\
\hline Age & Years & 1.04 & $<0.001$ \\
Diabetes duration & Years & 1.02 & 0.003 \\
Estimated glomerular & $10 \mathrm{~mL} / \mathrm{min} /$ & 0.90 & $<0.001$ \\
filtration rate & $1.73 \mathrm{~m}^{2}$ & & \\
Glycated hemoglobin & $\%$ & 1.02 & 0.405 \\
Serum creatinine & $\mathrm{mg} / \mathrm{dL}$ & 1.18 & $0.01 \mathrm{I}$ \\
Blood urea nitrogen & $10 \mathrm{mg} / \mathrm{dL}$ & 1.10 & $<0.001$ \\
Female gender & Dichotomous & 3.94 & $<0.001$ \\
Type 2 diabetes & Dichotomous & 1.73 & 0.014 \\
Retinopathy & Dichotomous & 1.28 & 0.051 \\
Chronic kidney disease & Dichotomous & 1.42 & 0.033 \\
Coronary artery disease & Dichotomous & 1.36 & 0.011 \\
Cerebrovascular disease & Dichotomous & 1.37 & 0.148 \\
Peripheral artery disease & Dichotomous & 1.01 & 0.921 \\
Somatic neuropathy & Dichotomous & 1.22 & 0.131 \\
Autonomic neuropathy & Dichotomous & 0.47 & 0.198 \\
\hline
\end{tabular}


Table 5 Risk factors associated with urinary tract infections multivariate analysis

\begin{tabular}{llll}
\hline Predictor & Unit & Odds ratio & $P$-value \\
\hline Age & Years & 1.04 & 0.001 \\
Diabetes duration & Years & 1.03 & 0.036 \\
Glycated hemoglobin & $\%$ & 1.13 & 0.017 \\
Blood urea nitrogen & $10 \mathrm{mg} / \mathrm{dL}$ & 1.10 & 0.023 \\
\hline
\end{tabular}

\section{Etiology of UTIs and AST}

Out of the 297 positive urine cultures, 284 (95.6\%) bacterial strains and 13 (4.4\%) Candida albicans strains were isolated. The gram-negative bacilli (GNB) from the Enterobacteriaceae family were predominant, with E. coli (209 strains [70.4\%]) being the most frequent, followed by Klebsiella pneumoniae (37 [12.5\%]), Proteus mirabilis (12 [4.0\%]), Enterobacter aerogenes (1 [0.3\%]), and Citrobacter freundii (1 [0.3\%]). The gram-positive cocci (GPC) were the second most frequently isolated germs. They included 15 (5.0\%) Enterococcus faecalis strains, 4 (1.4\%) Staphylococcus spp. strains, and $2(0.7 \%)$ Streptococcus agalactiae strains. The nonfermentative GNB were less present, with only $2(0.7 \%)$ strains of Pseudomonas aeruginosa and 1 (0.3\%) strain of Acinetobacter baumannii being isolated.

Regarding the antimicrobial susceptibility pattern (ASP) of the bacterial strains, we found an increased resistance of GNB to beta-lactam antibiotics. The penicillinase-producing strains were reported in $62.2 \%$ and $72.9 \%$ of the E. coli and $K$. pneumoniae isolates, respectively. The extended-spectrum beta-lactamase (ESBL)-producing strains were found in $4.3 \%$ and $10.8 \%$ of the E. coli and $K$. pneumoniae isolates, respectively. Furthermore, $1 P$. mirabilis and $1 P$. aeruginosa ESBL-producing strains were isolated. All ESBL-producing strains were multidrug-resistant (MDR), also showing resistance to other classes of antimicrobial agents (including quinolones, aminoglycosides, trimethoprim-sulfamethoxazole) and were isolated from patients with indwelling bladder catheters, with previous hospitalizations or prior antibiotic use. None of the isolates was reported to be carbapenem resistant. In GPC no unusual resistance patterns were recorded.

\section{Discussion}

This study found a prevalence of UTIs of $12.0 \%$ in patients with DM and, as expected, the prevalence was significantly higher in females $(17.7 \%)$ than in males $(5.2 \%)$. Various studies have estimated the prevalence of UTIs among patients with DM, obtaining similar results in the United States. ${ }^{5,11}$ In developing countries, the prevalence of UTIs is significantly higher: in Saudi patients with DM, Al-Rubeaan et al obtained a prevalence of UTIs of $25.3 \%,{ }^{12}$ higher in females $(41.1 \%)$ than in males (7.2\%) and similar in both types of DM, while in India, the reported prevalences of UTIs in patients with DM ranged between $42 \%$ and $45 \% .^{13,14}$ The variation in the reported rates of UTIs by geography may be due to cultural differences.

The presence of ASB is a major risk factor for developing symptomatic UTIs, ${ }^{15}$ the present study identifying ASB as the most prevalent type of UTI in patients with DM (9\%), with a prevalence similar to that reported in other previous studies. A meta-analysis of 22 studies regarding ASB in patients with DM has shown a prevalence of ASB of $12.2 \%$, higher in females $(14.2 \%)$ than in males $(2.3 \%),{ }^{16}$ similar to the present study's findings.

The diabetic patient's characteristics associated with the risk of developing UTIs were investigated and significant impact of age and diabetes duration was found, similar to the results reported by other studies. ${ }^{5,13,17,18}$ Longer duration of DM may lead to an increased prevalence of diabetic chronic complications, more frequent hospitalization, and catheterization of the urinary tract, all these conditions leading to an increased prevalence of UTIs. ${ }^{17}$

The univariate logistic regression analysis identified female gender and type $2 \mathrm{DM}$ as significant risk factors for UTIs, which is consistent with the findings of other studies. ${ }^{12,17}$ Also, the present study found that the presence of UTIs was associated with a significantly increased prevalence of chronic kidney disease and coronary artery disease. The association between chronic kidney disease and UTIs has been studied in the literature especially regarding the increased albumin excretion rate, with microalbuminuria or macroalbuminuria being incriminated as risk factors for the occurrence of UTIs. ${ }^{12,18}$ The association found in this study between the macrovascular complications of DM (coronary artery disease) and the risk of occurrence of UTIs was also demonstrated in other studies..$^{15,17}$

Glycemic control, defined by the HbAlc levels, had no significant influence on the risk for UTIs in the univariate analysis, but appeared as a significant risk factor when multivariate logistic regression model was applied. These findings are in agreement with those in previous reports suggesting an association between elevated $\mathrm{HbA} 1 \mathrm{c}$ levels and the presence of UTIs. ${ }^{19,20}$ Ribera et al found that a HbA1c level $>7 \%$ was associated with symptomatic UTIs in patients with DM, ${ }^{15}$ but this variable did not remain a risk factor when logistic regression analysis was applied. Other reports also have not found a correlation between metabolic control and the presence of UTIs. . $^{5,16-18,21}$ 
Although patients with DM may be more prone to UTIs caused by unusual and antibiotic-resistant microorganisms, this study identified most of the UTIs to be due to typical uropathogens. The bacteria associated with UTIs were predominantly E. coli (70.4\%) and other Enterobacteriaceae (17.1\%), findings similar to those from previous reports. ${ }^{5,13-15,17-20,22}$ There are studies reporting bacterial pathogens other than E. coli more often responsible for UTIs in patients with DM compared with patients without DM. Boyko et al ${ }^{5}$ identified Klebsiella and Enterococcus more often involved in ASB in females with DM versus females without DM.

The GNB recorded large variations of the resistance patterns to beta-lactam antibiotics, the antimicrobial agents frequently used in the treatment of UTIs. These values are close to those reported for Europe in the SENTRY study (2000). ${ }^{23}$

The ESBL-producing E. coli strains were found in $4.3 \%$ of the $E$. coli isolates, results similar with those reported by Sewify et al in a recent study from Kuwait. ${ }^{22}$ Other studies identified higher prevalences $(78.6 \%)$ of ESBLproducing $E$. coli strains in the urine cultures of patients with DM. ${ }^{20}$ Unlike the present findings regarding the ESBL-producing $K$. pneumoniae isolates, a previous report mentioned only $1.8 \%$ of the $K$. pneumoniae strains as being ESBL-producing. ${ }^{22}$ However, all the MDR ESBL-producing strains recorded in this study were isolated only in patients with indwelling bladder catheters, with previous hospitalizations or prior antibiotic use. Male gender, hospitalization, recurrent UTIs, and urinary catheterization were identified among the risk factors associated with UTIs caused by ESBLproducing enterobacteria. ${ }^{24}$

A major advantage of this study is that it reflects the influence of several host factors on the risk of developing UTIs in patients with DM. In Romania, data regarding infections in patients with DM are poor, with only a few studies being published. The findings are similar to those obtained by other studies and they complete the existing results in this country.

The limitations of the study include its retrospective chart review nature. In addition, the fact that it only included hospitalized patients led to a limited generalization of the findings, which cannot be extrapolated to the entire population of DM patients. The decreased prevalence of lower UTIs obtained in this study may be because this condition is usually treated in ambulatory conditions, not representing a hospitalization reason for patients with DM.

\section{Conclusion}

A $12.0 \%$ prevalence of UTIs was observed in this large population of hospitalized adult patients with DM. Age, diabetes duration, and metabolic control (HbA1c levels) were independent risk factors for the occurrence of UTIs. $E$. coli is the most frequent pathogen responsible for UTIs in patients with DM, followed by $K$. pneumoniae and E. faecalis. The results obtained in this study have a great practicability because they raise a warning signal regarding UTIs in patients with DM and deliver important information for the evaluation of the global risk of patients with DM and infection, in order to initiate the adequate treatment. Monitoring the ASP of isolated microorganisms provides rational use of antimicrobial agents for the treatment of UTIs, avoiding the development of antibiotic-resistant urinary pathogens.

\section{Disclosure}

The authors report no conflicts of interest in this work.

\section{References}

1. Shah BR, Hux JE. Quantifying the risk of infectious diseases for people with diabetes. Diabetes Care. 2003;26(2):510-513.

2. Schappert SM. Ambulatory care visits to physician offices, hospital outpatient departments, and emergency departments: United States, 1997. National Center for Health Statistics. Vital Health Stat. 1999; 13(143):1-39.

3. Foxman B. Epidemiology of urinary tract infections: incidence, morbidity and economic costs. Am J Med. 2002;113(Suppl 1A):5S-13S.

4. Boyko EJ, Lipsky BA. Infection in diabetes. In: National Diabetes Data Group, editor. Diabetes in America. 2nd ed. Bethesda, MA: Harris; 1995:485-499.

5. Boyko EJ, Fihn SD, Scholes D, Abraham L, Monsey B. Risk of urinary tract infection and asymptomatic bacteriuria among diabetic and nondiabetic postmenopausal women. Am J Epidemiol. 2005;161(6): $557-564$.

6. Fünfstück R, Nicolle LE, Hanefeld M, Naber KG. Urinary tract infection in patients with diabetes mellitus. Clin Nephrol. 2012;77(1):40-48.

7. Nitzan O, Elias M, Chazan B, Saliba W. Urinary tract infections in patients with type 2 diabetes mellitus: review of prevalence, diagnosis, and management. Diabetes Metab Syndr Obes. 2015;8:129-136.

8. Stapleton A. Urinary tract infections in patients with diabetes. Am J Med. 2002;113(Suppl 1A):80S-84S.

9. Bonadio M, Costarelli S, Morelli G, Tartaglia T. The influence of diabetes mellitus on the spectrum of uropathogens and the antimicrobial resistance in elderly adult patients with urinary tract infection. $B M C$ Infect Dis. 2006;6:54.

10. Clinical and Laboratory Standards Institute. Performance Standards for Antimicrobial Susceptibility Testing. Twentieth Informational Supplement. M100-S20. Wayne, PA: Clinical and Laboratory Standards Institute; 2010.

11. Grandy S, Fox KM, Hardy E. Prevalence and recurrence of urinary tract and genital infections among adults with and without type 2 diabetes mellitus in the general population: a longitudinal cohort study. J Diabetes Res Clin Metab. 2013;2(5):8.

12. Al-Rubeaan KA, Moharram O, Al-Naqeb D, Hassan A, Rafiullah MR. Prevalence of urinary tract infection and risk factors among Saudi patients with diabetes. World J Urol. 2013;31(3):573-578.

13. Janifer J, Geethalakshmi S, Satyavani K, Viswanathan V. Prevalence of lower urinary tract infection in South Indian type 2 diabetic subjects. Indian J Nephrol. 2009;19(3):107-111.

14. Ramana BV, Chaudhury A. Prevalence of uropathogens in diabetic patients and their resistance pattern at a tertiary care centre in south India. Int J Biol Med Res. 2012;3(1):1433-1435. 
15. Ribera MC, Pascual R, Orozco D, Pérez Barba C, Pedrera V, Gil V. Incidence and risk factors associated with urinary tract infection in diabetic patients with and without asymptomatic bacteriuria. Eur J Clin Microbiol Infect Dis. 2006;25(6):389-393.

16. Renko M, Tapanainen P, Tossavainen P, Pokka T, Uhari M. Metaanalysis of the significance of asymptomatic bacteriuria in diabetes. Diabetes Care. 2011;34(1):230-235.

17. Zhanel GG, Nicolle LE, Harding GK; for Manitoba diabetic urinary infection Study Group. Prevalence of asymptomatic bacteriuria and associated host factors in women with diabetes mellitus. Clin Infect Dis. 1995;21(2):316-322.

18. Geerlings SE, Stolk RP, Camps MJ, et al. Asymptomatic bacteriuria may be considered a complication in women with diabetes. Diabetes Care. 2000;23(6):744-749.

19. Bonadio M, Boldrini E, Forotti G, et al. Asymptomatic bacteriuria in women with diabetes: influence of metabolic control. Clin Infect Dis. 2004;38(6):e41-e45.

20. Aswani SM, Chandrashekar UK, Shivashankara KN, Pruthvi BC. Clinical profile of urinary tract infections in diabetics and non-diabetics. Australas Med J. 2014;7(1):29-34.
21. Geerlings SE, Stolk RP, Camps MJ, Netten PM, Collet TJ, Hoepelman AI. Risk factors for symptomatic urinary tract infection in women with diabetes. Diabetes Care. 2000;23(12):1737-1741.

22. Sewify M, Nair S, Warsame S, et al. Prevalence of urinary tract infection and antimicrobial susceptibility among diabetic patients with controlled and uncontrolled glycemia in Kuwait. J Diabetes Res. 2016;2016: 6573215.

23. Gordon KA, Jones RN; for SENTRY Participant Groups (Europe, Latin America, North America). Susceptibility patterns of orally administered antimicrobials among urinary tract infection pathogens from hospitalized patients in North America: comparison report to Europe and Latin America: results from the SENTRY Antimicrobial Surveillance Program (2000). Diagn Microbiol Infect Dis. 2003;45(4):295-301.

24. Briongos-Figuero LS, Gomez-Traveso T, Bachiller-Luque P, et al. Epidemiology, risk factors and comorbidity for urinary tract infections caused by extended-spectrum beta-lactamase (ESBL)-producing enterobacteria. Int J Clin Pract. 2012;66(9):891-896.
Therapeutics and Clinical Risk Management

\section{Publish your work in this journal}

Therapeutics and Clinical Risk Management is an international, peerreviewed journal of clinical therapeutics and risk management, focusing on concise rapid reporting of clinical studies in all therapeutic areas, outcomes, safety, and programs for the effective, safe, and sustained use of medicines. This journal is indexed on PubMed Central, CAS,

\section{Dovepress}

EMBase, Scopus and the Elsevier Bibliographic databases. The manuscript management system is completely online and includes a very quick and fair peer-review system, which is all easy to use. Visit http://www.dovepress.com/testimonials.php to read real quotes from published authors.

Submit your manuscript here: http://www.dovepress.com/therapeutics-and-clinical-risk-management-journal 